\title{
MECHANISM DESIGN AND MOTION PLANNING OF PARALLEL-CHAIN NONHOLONOMIC MANIPULATOR
}

\author{
Li, L. \\ School of Mechanical Engineering, Baoji University of Arts and Sciences, Baoji 721016, China \\ E-Mail: leeliang@126.com
}

\begin{abstract}
Inspired by the nonholonomic theory, this paper proposes a parallel-chain nonholonomic manipulator with a chainable kinetics model. To build the manipulator, the friction disc motion synthesis and decomposition mechanism was taken as the joint transmission component. Based on Chow's theorem, the kinetics model of the manipulator was proved as nonholonomic and controllable. Then, the system's configuration coordinates were mapped from the joint space to the chain space via coordinate transformation, and the manipulator motion was planned in the chain space. Through two simulation experiments, it is proved that all joints of the proposed manipulator can move to the target configuration within the specified time. To sum up, the author successfully built an underactuated manipulator that can drive the motion of four joints with two motors. The research findings lay the basis for the development of small lightweight manipulators.

(Received, processed and accepted by the Chinese Representative Office.)
\end{abstract}

Key Words: Nonholonomic, Parallel-Chain, Chain Transformation, Motion Planning

\section{INTRODUCTION}

In analytical mechanics, a nonholonomic system refers to a system whose constraint equations contain the derivative of the coordinates with respect to time. In other words, the system speed or acceleration is under constraint. The nonholonomic mechanical system is underactuated, as it has fewer degrees of freedom (DoFs) than the number of dimensions in its configuration space. Hence, a multi-dimensional motion in the configuration space can be determined by a few control inputs, making it possible to design compact, lightweight multijoint manipulators. The research into nonholonomic manipulator carries practical implications for the development of assistive robots like small robots, medical robots and multi-fingered dexterous hands.

In the field of robotics, the research into nonholonomic system mainly concentrates on the control of existing nonholonomic robots, such as wheeled mobile robots, spherical robots and underwater robots [1-3]. Owing to the motion nonlinearity of nonholonomic robots, it is necessary to develop a unique path planning method for each nonholonomic system, adding to the difficulty in the motion control of new nonholonomic robots.

In reality, many kinematics models of existing nonholonomic robots (e.g. wheeled mobile robots and trailer systems) can be converted into the chained model, a drift-free controllable nonholonomic system model. A system whose kinematics equations can be described with a chained model is called a chained system. Such a system boasts excellent properties (nilpotent and smooth), and simple structured mathematical model. In view of these advantages, many scholars have created nonholonomic robots with chainable kinematics model. For example, Nakamura proposed an underactuated manipulator based on a friction ball vector synthesis and decomposition mechanism [4]. The manipulator supports path planning via the control method of a chained system, as its kinematics model can be converted into a chained model. Under the diffeomorphism of chained transformation, paper [5] designs the gear steering connection mechanism for nonpowered trailer, and constructs a chainable wheeled mobile trailer system that can accurately track the target trajectory. Yamaguchi developed a 4 DoFs 
wheeled mobile robot capable of chained transformation [6-8]; the wheeled mobile mechanism is controlled precisely with the drive angle and azimuth of the traction robot and the angle of the active steering system mounted on the connecting rod.

Based on the previous research into a parallel-chain type chainable nonholonomic manipulator [9-11], this paper puts forward a two-motor parallel-chain four-joint nonholonomic manipulator. In the parallel-chain manipulator, the friction disc motion synthesis and decomposition mechanism serve as the joint transmission component, and the motion is transferred by dual universal joint in parallel-chain mode. Compared to the parallelchain manipulator, the proposed manipulator, with a concise structure and a small power loss, offers an effective solution to the conflict between the number of drive units and manipulator mass in multi-joint manipulator.

The remainder of this paper is organized as follows: Section 2 introduces the design of the parallel-chain nonholonomic manipulator; Section 3 establishes the kinematics model of the manipulator, demonstrates the manipulator controllability, and analyses the chain transformation features; Section 4 plans a path that maps back to the joint space in the chain space based on the control law of time polynomial motion planning; Section 5 concludes that the proposed manipulator can move from the initial configuration to the target configuration within the specific time under the control law of the chained system, and outperforms the parallel-chain manipulator in trajectory simplicity and motion efficiency.

\section{PARALLEL-CHAIN NONHOLONOMIC MANIPULATOR MECHANISM}

\subsection{Motion principle of friction disc}

As shown in Fig. 1, when the friction wheel with the radius $r$ rotates around axis $I$ at the angular velocity $W_{i}$, there is only pure rolling between the friction wheel and the friction disc; then, the friction disc will rotate around axis $O$ at the angular velocity $W_{0}$. The friction wheel and the friction disc are perpendicular to each other. Let $M$ be the contact point between the friction wheel and the friction disc. The friction wheel can also rotate relative to the friction disc around the connecting line between its own axis and point $M$. When the rotation angle reaches $\alpha$, the linear velocities of the friction wheel and the friction disc were plotted into a vector diagram (Fig. 1 b).

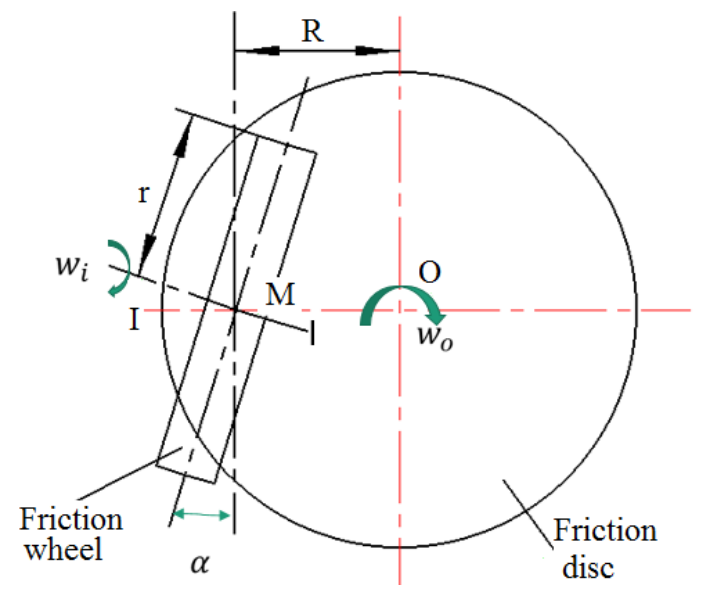

a)

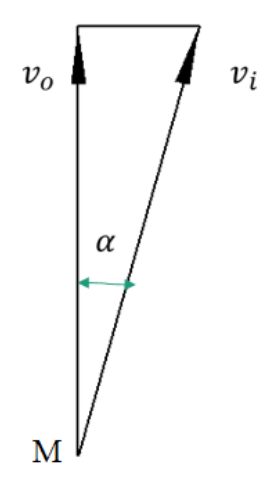

b)

Figure 1: Friction disc motion synthesis and decomposition mechanism.

Then, the following equation holds: $V_{o}=W_{o} R=V_{i} \cos \alpha=W_{i} r \cos \alpha$. 
Thus, we have:

$$
W_{o}=\frac{r}{R} w_{i} \cos \alpha
$$

where $R$ is the distance between point $M$ and the centre of friction disc; $V_{i}$ and $V_{o}$ are the linear velocities of the friction wheel and the friction disc at point $M$, respectively.

It can be seen that the transmission ratio between the friction wheel and the friction disc can be controlled by adjusting the angle $\alpha$. Hence, $\alpha$ was defined as the transmission angle.

The rolling-induced relative motion of the friction wheel on the friction disc depends on the relative change of configuration. Based on the relative configuration-variable structure, the designed friction disc motion synthesis and decomposition mechanism is subjected to the nonholonomic constraint [12-15].

\subsection{Design of parallel-chain nonholonomic manipulator}

A friction disc mechanism was arranged at each joint of the manipulator. In the mechanism, the friction wheel and the friction disc are permanently connected to the front and rear joints, respectively. The transmission ratio between the two components changes with the included angle between them (i.e. the joint angle). Fig. 2 illustrates the structure of parallel-chain fourjoint manipulator.

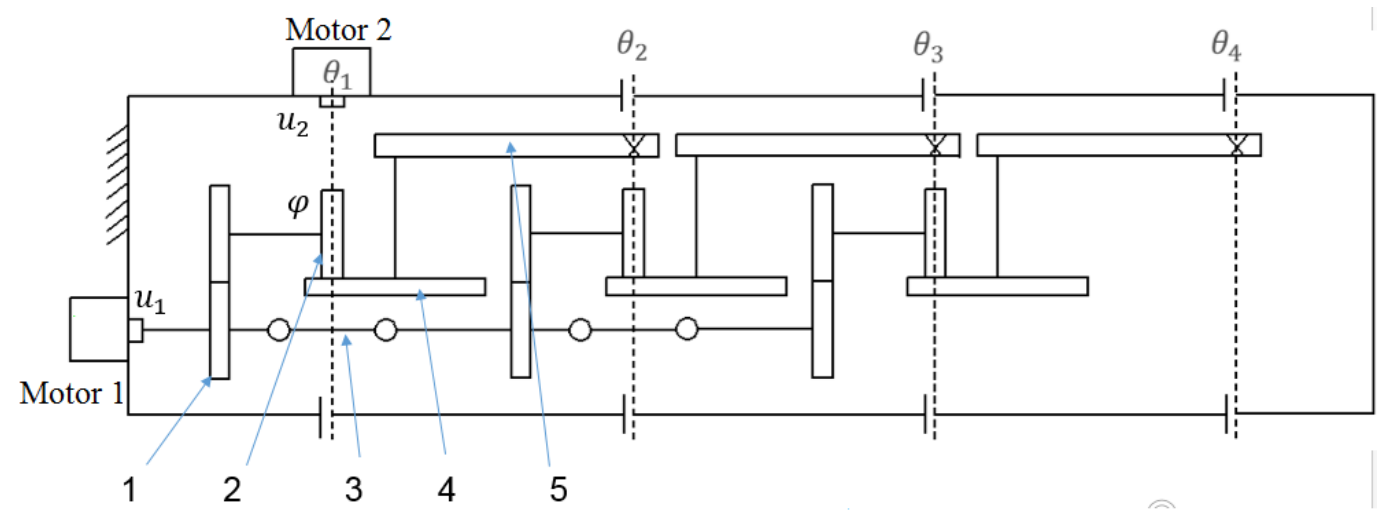

1. Gear, 2. Friction wheel, 3. Dual universal joint, 4. Friction plate, 5. Synchronous belt

Figure 2: Mechanism of parallel-chain four-joint manipulator.

The rotation of motor 2 directly drives joint 1 to rotate about the axis by the angle $\theta_{1}$. Since friction wheel 1 is fixed to the frame through the side plate and friction plate 1 is fixed to the first joint, motor 2 controls the rotation angle $\theta_{1}$ of joint 1 as if a transmission angle $\theta_{1}$ is added to the friction transmission of the friction wheel and the friction disc.

Motor 1 transmits its energy in two directions. In one direction, the motor drives the friction wheel through gears, the friction wheel drives the friction disc via rolling friction, and the friction disc drives joint 2 to rotate by the angle $\theta_{2}$ through the synchronous belt; meanwhile, the motor adds a transmission angle $\theta_{2}$ between the friction wheel and the friction disc at joint 2. In the other direction, motor 1 transmits its energy to the nearest rear joint via the dual universal joint, so that each rear joint can transmit energy to its next rear joint in turns.

In this way, the four joints can be driven by two motors. The prototype of the parallelchain four-joint manipulator is presented in Fig. 3. 


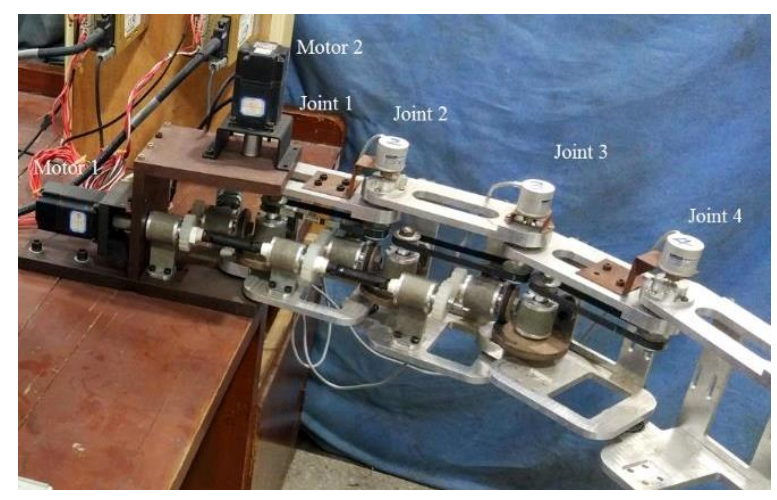

Figure 3: Prototype of parallel-chain four-joint manipulator.

The following issues call for special attention in the production and assembly of the prototype:

(1) To ensure effective, reliable and accurate transmission of motion and force, there should be sufficient friction between the friction wheel and the friction disc. Hence, the material should have a large friction coefficient. Besides, a certain amount of positive pressure should be applied to point $\mathrm{M}$, such that there is no relative sliding but pure rolling between the friction wheel and the friction.

(2) As shown in Fig. 4 a, point M should be placed on the axis of the joint. Otherwise, the friction wheel will slide on the friction disc when the joint rotates to a certain angle. The resulting change in the distance $R$ between point $\mathrm{M}$ and the centre of the friction disc will reduce the transmission accuracy.

(3) The input shaft and the output shaft of the dual universal joint should have the same rotational angular velocity. In other words, the centreline $\mathrm{OO}$ of the dual universal joint must be consistent with the joint axis. Moreover, the intermediate shaft should be retractable, so as to compensate for the change in the axial distance between the input and output shafts caused by the rotation of manipulator joints (Fig. 4 b).

(4) For the compactness and lightweight of the whole structure, the periphery of the connecting rod should be made into large rounded corner and the central part of the rod should be grooved, without sacrificing the strength and rigidity. In the horizontal direction, the main energy transmission chain (dual universal joint) and the motion transmission chain (friction wheel and friction disc) should be arranged at the same distance from the edge of the manipulator. The distance should approximate the spacing between the two transmission chains. In the vertical direction, the two transmission chains should be placed symmetrically about the connecting rod. All these arrangements ensure that the centre of mass of the manipulator is close to its geometric centre, thereby improving the kinetic performance of the manipulator.

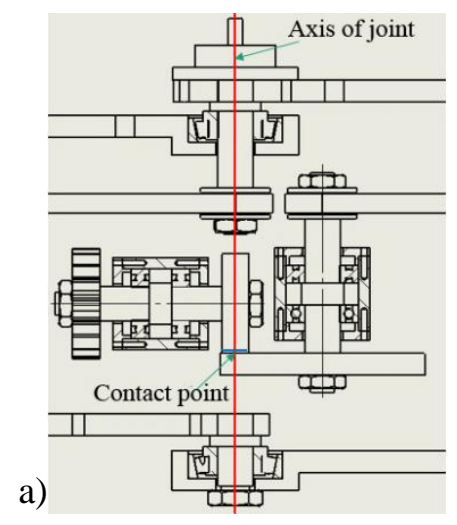

b) Input shaft

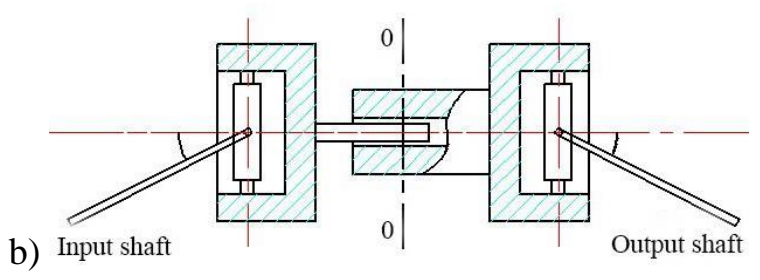

Figure 4: a) location of point $\mathrm{M}, \mathrm{b}$ ) structure of dual universal joint. 


\section{KINEMATICS ANALYSIS AND CHAIN TRANSFORMATION}

\subsection{Kinematics modelling}

The configuration space of the four-joint nonholonomic manipulator hinges on the joint rotation angle $\theta_{i}(i=1,2,3,4)$ and the angular displacement $\varphi$ of the friction wheel. Hence, the generalized coordinate vector of the manipulator system was defined as $q=\left[q_{1}, q_{2}, q_{3}, q_{4}, q_{5}\right]=\left[\varphi, \theta_{1}, \theta_{2}, \theta_{3}, \theta_{4}\right]$, and the control inputs as the angular velocities of the two motors $u_{1}$ and $u_{2}$. According to the kinematics relationship, the kinematics model of the parallel-chain four-joint manipulator can be derived as:

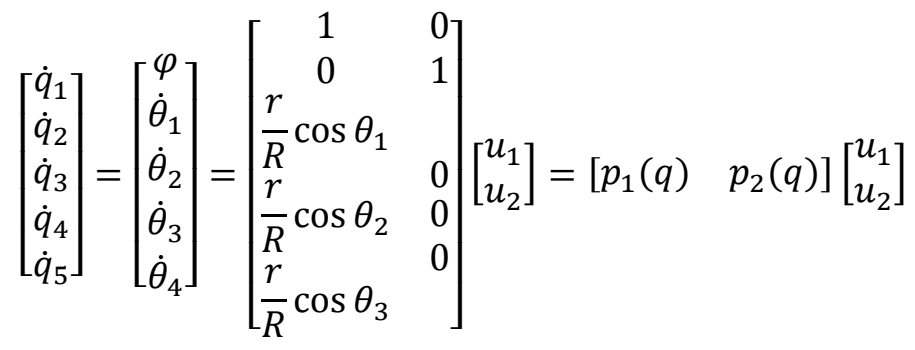

where $r$ is the radius of the friction wheel.

\subsection{Controllability analysis}

Eq. (2) describes a drift-free control system. For such a drift-free symmetric affine system, the reachable space is expanded from the distribution $\Delta(q)=\operatorname{span}\left\{p_{1}, p_{2}\right\}$.

According to the controllability conditions of nonholonomic systems (Chow's theorem) [16], a drift-free affine system is controllable if its reachable distribution $\Delta_{p}(q)=$ $\operatorname{span}\left\{p_{1}, p_{2},\left[p_{1}, p_{2}\right],\left[p_{1},\left[p_{1}, p_{2}\right]\right], \ldots\right\}$ is in full rank. Note that $\left[p_{1}, p_{2}\right]$ and $\left[p_{1},\left[p_{1}, p_{2}\right]\right]$ are the Lie bracket operations on vectors $p_{1}, p_{2}$ and $p_{1},\left[p_{1}, p_{2}\right]$, respectively. Then, we have $\left[p_{1}, p_{2}\right]=\frac{\partial p_{2} q}{\partial q} p_{1}(q)-\frac{\partial p_{1}(q)}{\partial q} p_{2}(q)$.

Thus, the reachable space of the parallel-chain nonholonomic four-joint manipulator can be expressed as:

$$
\begin{gathered}
\Delta_{p}(q)=\operatorname{span}\left\{p_{1}, p_{2},\left[p_{1}, p_{2}\right],\left[p_{1},\left[p_{1}, p_{2}\right]\right],\left[p_{1},\left[p_{1},\left[p_{1}, p_{2}\right]\right]\right]\right\}= \\
\left.\qquad \begin{array}{ccccc}
1 & 0 & 0 & 0 & 0 \\
0 & 1 & 0 & 0 & 0 \\
k c_{1} & 0 & k s_{1} & 0 & 0 \\
k c_{2} & 0 & 0 & k^{2} s_{1} s_{2} & k^{3} s_{1} c_{1} c_{2} \\
k c_{3} & 0 & 0 & 0 & k^{3} s_{1} s_{2} s_{3}
\end{array}\right]
\end{gathered}
$$

where $k=\frac{r}{R}, c_{i}=\cos \theta_{i}, s_{i}=\sin \theta_{i} \neq 0(i=1,2,3)$.

It can be derived from Eq. (3) that $\operatorname{dim} \Delta_{p}(q)=5$ if $\sin \theta_{1} \neq 0, \sin \theta_{2} \neq 0$ and $\sin \theta_{3} \neq 0$, that is, $\sin \theta_{i} \neq 0(i=1,2,3)$. In this case, the rank of the matrix equals the number of dimensions in the configuration space. In other words, the reachable space expanded from the system is involutive, which satisfies the controllability rank condition. Therefore, the parallel-chain four-joint nonholonomic manipulator is nonholonomic and controllable in the fivedimensional reachable space, as long as its work space satisfies $\theta_{i} \neq 0(i=1,2,3)$. In this case, the motion of the five configuration variables can be controlled with two motors.

\subsection{Analysis of chain transformation features}

After investigating a wheeled mobile robot system with $n$ trailers, Sørdalen proposed the conditions and methods for the chain transformation of a drift-free affine system with a triangular configuration [17], similar to Eq. (2): 


$$
\left\{\begin{array}{c}
\dot{q}_{1}=u_{1} \\
\dot{q}_{2}=u_{2} \\
\dot{q}_{1}=f_{i}\left(\underline{q_{i-1}}\right) u_{1}
\end{array} i \in\{3, \ldots, n\} .\right.
$$

If the smooth function $f_{i}\left(\underline{q}_{i-1}\right)$ satisfies $\left|\frac{\partial f_{i}\left(\underline{q_{i-1}}\right)}{\partial q_{i-1}}\right|_{q=q_{0}} \neq 0\left(\forall_{i} \in\{3,4, \ldots, n\}\right)$ in the neighbourhood of $q_{0}$, there exist diffeomorphic coordinate transformation and input transformation such that the system can be converted to a chained system.

If $\theta_{i} \neq 0(i=1,2,3)$, then the chain transformation and input feedback transformation of the four-joint nonholonomic manipulator can be expressed as:

$$
\begin{gathered}
\left\{\begin{array}{c}
Z_{5}=\theta_{4} \\
Z_{4}=k \cos \theta_{3} \\
Z_{2}=k^{3}\left(k^{2} \cos \theta_{2} \sin \theta_{3} \sin \theta_{2} \sin \theta_{3}-\cos ^{2} \theta_{2} \cos \theta_{3}\right) \\
Z_{1}=\varphi
\end{array}\right. \\
\left\{\begin{array}{c}
v_{1}=\dot{z}_{1}=\dot{\varphi}=u_{1} \\
v_{2}=\dot{z}_{2}=k^{4} c_{2}\left(3 c_{1} s_{2} s_{3}+s_{3} c_{2}^{2}+s_{3} c_{2}^{2}\right) u_{1}-k^{3} s_{1} s_{2} s_{3} u_{2}
\end{array}\right.
\end{gathered}
$$

\section{MOTION PLANNING FOR PARALLEL-CHAIN FOUR-JOINT NONHOLONOMIC MANIPULATOR}

The basic idea of the motion planning for chainable nonholonomic manipulator is to map the initial configuration $q^{i}$ and target configuration $q^{f}$ of the system into the initial configuration $z^{i}$ and target configuration $z^{f}$ of the chain space, forming a path from the initial configuration $z^{i}$ to the target configuration $z^{f}$, and then map the path to the joint space through inverse chain transformation.

The relative mature motion planning methods for chained systems include piecewise constant input method, trigonometric function input method, polynomial input method, and switching control method. Among them, the polynomial input method stands out for its simple integration operation and the ability to control all variables to move to the target configuration along a smooth trajectory. The polynomial expression of the time-variation of two control inputs is:

$$
\left\{\begin{array}{c}
V_{1}(t)=b_{1} \\
V_{2}(t)=b_{2}+b_{3} t+b_{4} t^{2}
\end{array}\right.
$$

The motion planning aims to find a bounded control input $u(t)$ such that the system reaches the target configuration $z^{f}$ from the initial configuration $z^{i}$ over the specified time $T$. In other words, the system satisfies the following constraints:

$$
\left\{\begin{array}{l}
f_{1}=Z_{2}(T)-Z_{2}^{f}=0 \\
f_{2}=Z_{3}(T)-Z_{3}^{f}=0 \\
f_{3}=Z_{4}(T)-Z_{4}^{f}=0 \\
f_{4}=Z_{5}(T)-Z_{5}^{f}=0
\end{array}\right.
$$

Through integration, the chained system can be expressed as: 


$$
\left\{\begin{array}{c}
z_{2}(T)=b_{2} T+\frac{T^{2}}{2} b_{3}+\frac{T^{3}}{3} b_{4}+z_{2}^{i} \\
z_{3}(T)=\frac{T^{2}}{2} b_{1} b_{2}+\frac{T^{3}}{6} b_{1} b_{3}+\frac{T^{4}}{12} b_{1} b_{4}+T z_{2}^{i} b_{1}+z_{3}^{i} \\
z_{4}(T)=\frac{T^{3}}{6} b_{1}^{2} b_{2}+\frac{T^{4}}{24} b_{1}^{2} b_{3}+\frac{T^{5}}{60} b_{1}^{2} b_{4}+\frac{T^{2}}{2} b_{1}^{2} z_{2}^{i}+z_{3}^{i} T b_{1}+z_{4}^{i} \\
z_{5}(T)=\frac{T^{4}}{24} b_{1}^{3} b_{2}+\frac{T^{5}}{120} b_{1}^{3} b_{3}+\frac{T^{6}}{360} b_{1}^{3} b_{4}+\frac{T^{3}}{6} b_{1}^{3} z_{2}^{i}+\frac{T^{2}}{2} z_{3}^{i} b_{1}^{2}+T z_{4}^{i} b_{1}+z_{5}^{i}
\end{array}\right.
$$

Substituting Eq. (8) into Eq. (7), we have a set of nonlinear equations about $b_{1}, b_{2}, b_{3}$ and $b_{4}$. The Newton iteration form of the equation set is:

$$
b^{(k+1)}=b^{(k)}-\left[f^{\prime}\left(b^{(k)}\right)\right]^{+} F\left(b^{(k)}\right)
$$

where $F^{\prime}(b)$ is the Jacobian matrix of $F(b) ;\left[F^{\prime}(b)\right]^{+}$is the pseudo-inverse of $F^{\prime}(b)$. Let $b=\left[b_{1}, b_{2}, b_{3}, b_{4}\right]^{T}$ and $F=\left[f_{1}, f_{2}, f_{3}, f_{4}\right]^{T}$.

Given the initial value $b^{(0)}, b$ can be calculated by the iteration Eq. (9). Then, the trajectory of $z_{i}^{(t)}$ can be acquired by substituting $b$ into Eq. (8). Through the inverse chain transformation of Eq. (4), we can obtain the expression of the angular displacement of each joint with respect to the $z$-variable. Thus, the motion curves of the angular displacement of the four joints can be expressed as:

$$
\left\{\begin{array}{c}
\theta_{4}=Z_{5} \\
\theta_{3}=\operatorname{arcos}\left(Z_{4} / K\right) \\
\theta_{2}=\operatorname{arcos}\left(-\frac{Z_{3}}{K^{2} \sin \theta_{3}}\right) \\
\theta_{1}=\operatorname{arcos}\left(\frac{\frac{Z_{2}}{K^{3}}+\cos \theta_{3}\left(\cos \theta_{2}\right)^{2}}{\sin \theta_{2} \sin \theta_{3}}\right)
\end{array}\right.
$$

\section{SIMULATION EXPERIMENTS}

\section{Experiment 1:}

Let the initial configuration $\theta^{i}=\left[\begin{array}{llll}\theta_{1}^{i} & \theta_{2}^{i} & \theta_{3}^{i} & \theta_{4}^{i}\end{array}\right]^{T}$ of an parallel-chain four-joint nonholonomic manipulator be $\left[5^{0} 5^{0} 5^{0} 5^{0}\right]^{T}$ and the target configuration of that manipulator be $\theta^{f}=\left[\begin{array}{llll}\theta_{1}^{f} & \theta_{2}^{f} & \theta_{3}^{f} & \theta_{4}^{f}\end{array}\right]^{T}=\left[\begin{array}{llll}15^{0} & 15^{0} & 15^{0} & 15^{0}\end{array}\right]^{T}$.

Substituting the configurations into Eq. (4), the boundary conditions in the chain space can be derived as $z^{i}=\left[\begin{array}{llll}z_{1}^{i} & z_{2}^{i} & z_{3}^{i} & z_{4}^{i}\end{array}\right]^{T}=\left[\begin{array}{llll}-0.1958 & -0.0297 & 0.5822 & 0.0873\end{array}\right]^{T}$ and $z^{f}=$ $\left[\begin{array}{llll}z_{1}^{f} & z_{2}^{f} & z_{3}^{f} & z_{4}^{f}\end{array}\right]^{T}=\left[\begin{array}{llll}-0.1670 & -0.0854 & 0.5645 & 0.2618\end{array}\right]^{T}$.

a)

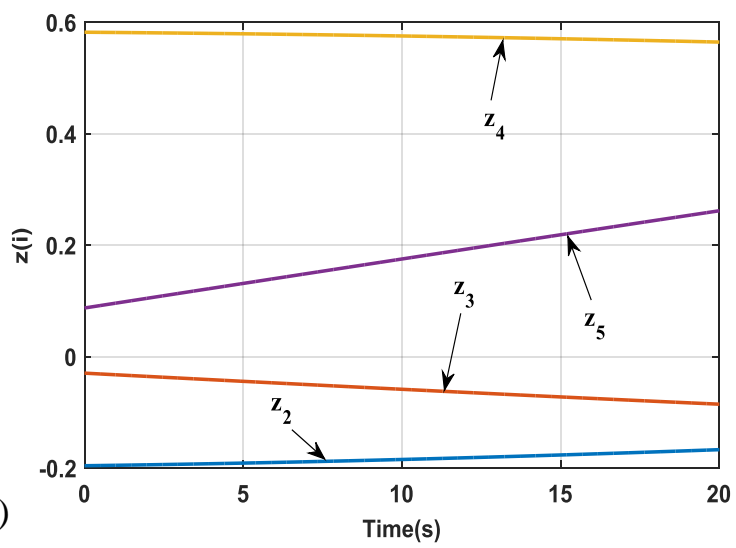

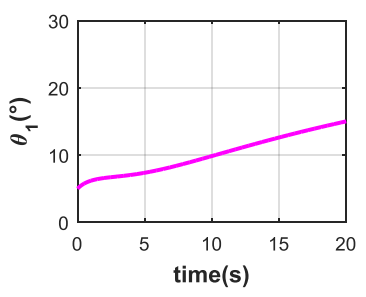
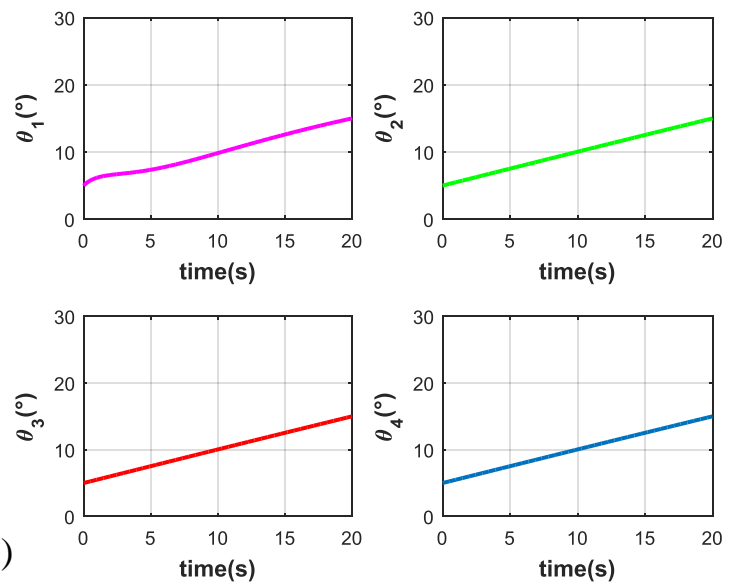

Figure 5: Trajectory, a) of variable $z$ in the chain space, b) of each joint in the joint space. 
Let the motion time $T=20 \mathrm{~s}$ and $b^{(0)}=\left[\begin{array}{llll}0.1 & 0.1 & 0.1 & 0.1\end{array}\right]^{T}$. The termination condition of the system iteration was set with the error at the termination time:

$\mathrm{e}=\sqrt{\left(z_{2}(T)-Z_{2}^{g}\right)^{2}+\left(z_{3}(T)-Z_{3}^{g}\right)^{2}+\left(z_{4}(T)-Z_{4}^{g}\right)^{2}+\left(z_{5}(T)-z_{5}^{g}\right)^{2}}<10^{-6}$. Then, Eq. (9) was solved by Newton iteration method. Through 9 iterations, we have $b=\left[\begin{array}{lll}b_{1} & b_{2} & b_{3} \\ b_{4}\end{array}\right]^{\mathrm{T}}=$ $\left[\begin{array}{llll}0.0151831 & 0.0007583 & 0.0000772 & -0.0000007\end{array}\right]^{T}$. Substituting $b$ into Eq. (8), we have the time-variation curve of variable $z$ (Fig. 5 a). According to Eq. (10), the path in the chain space can be mapped back to the joint space via inverse transformation. Under the time polynomial input control, the output of the four joints of the nonholonomic manipulator is as shown in Fig. $5 \mathrm{~b}$.

At $T=20 \mathrm{~s}, \theta_{1}=14.99999999^{\circ}, \theta_{2}=14.99999999^{\circ}, \theta_{3}=14.99999999^{\circ}$ and $\theta_{4}=14.99999999^{\circ}$.

Let the error of target configuration be: $\mathrm{e}=\frac{\theta^{r}-\theta^{g}}{\theta^{g}-\theta^{i}}$

where $\theta^{r}$ is the actual displacement of joint rotation. At this time, target configuration error of each joint is $\mathrm{e}_{\theta_{1}}=0.0000001 \%, \mathrm{e}_{\theta_{2}}=0.0000001 \%, \mathrm{e}_{\theta_{3}}=0.0000001 \%$ and $\mathrm{e}_{\theta_{4}}=0.0000001 \%$. The simulation results show that, under the time polynomial input control, all joints have smooth trajectories except for a slight fluctuation of joint 1 in the initial phase, and arrive at the target configuration.

\section{Experiment 2:}

Let the initial configuration of the proposed manipulator $\theta^{i}=\left[\begin{array}{llll}\theta_{1}^{i} & \theta_{2}^{i} & \theta_{3}^{i} & \theta_{4}^{i}\end{array}\right]^{T}$ be $\left[\begin{array}{llll}20^{0} & 20^{0} & 20^{0} & 20^{0}\end{array}\right]^{T}$ and its target configuration be $\theta^{f}=\left[\begin{array}{llll}\theta_{1}^{f} & \theta_{2}^{f} & \theta_{3}^{f} & \theta_{4}^{f}\end{array}\right]^{T}=\left[\begin{array}{llll}10^{0} & 10^{0} & 10^{0} & 10^{0}\end{array}\right]^{T}$. Suppose the motion time $T=20 \mathrm{~s}$. Through simulation, the time-variation trajectories of the chain variable and joint variable are as shown in Figs. 6 a and 6 b, respectively.

a)
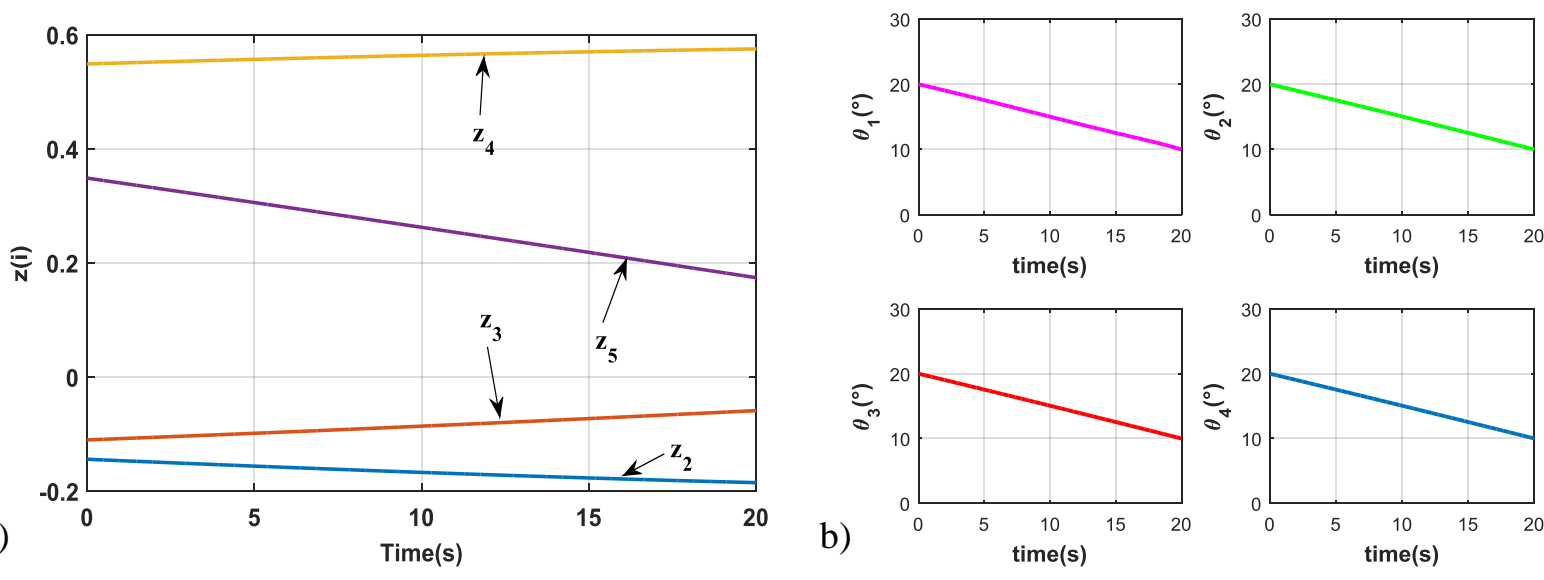

Figure 6: Trajectory, a) of variable $z$ in the chain space, b) of each joint in the joint space.

At $T=20 \mathrm{~s}, \theta_{1}=10.000000000016^{\circ}, \theta_{2}=10.000000000016^{\circ}, \theta_{3}=10^{\circ}$ and $\theta_{4}=10^{0}$. The simulation results show that each joint of the manipulator has a smooth trajectory and arrives at the target configuration within the specified time.

Comparing the results of the two simulation experiments, it is clear that, all joints of the parallel-chain four-joint manipulator can move accurately from the initial configuration to the target configuration within the specified time, when the input is controlled by the time polynomial obtained through Newton iteration. The motion of each joint is stable, with virtually no large fluctuation. Therefore, the Newton iteration-based polynomial input control is a feasible motion planning method for the parallel-chain four-joint nonholonomic manipulator. 


\section{CONCLUSIONS}

Considering the friction disc motion synthesis and decomposition mechanism, this paper proposes a chainable-type parallel-chain four-joint nonholonomic manipulator based on the parallel-chain nonholonomic manipulator. According to the nonlinear control theory, the author proved that the reachable space expanded from the manipulator system satisfies the involution distribution, i.e. the system is controllable. Then, the nonholonomic motion planning was transformed into the solution to nonlinear equation set, using the time polynomial input method of the chained system. The unknown coefficients of the time polynomial were solved by Newton iteration method. After that, two simulation experiments were performed on the motion between initial and target configurations. The results show that all joints of the proposed manipulator can move stably and accurately from the initial configuration to the target configuration within the specified time.

Nevertheless, there is no guarantee that the planned path between the initial configuration and the target configuration in the chain space can be transformed back into the joint space without singularity, especially when the joint variables are coupled tightly due to the increase in the number of joints on the manipulator. Thus, the key to the path planning of nonholonomic manipulator lies in the existence of the solution to inverse transformation of the planed path from the chain space to joint space. In the future research, the author will construct the mathematical expression of the geometric and topological features of the nonholonomic path, identify the conditions for the path between adjacent configurations to converge into the chain space, and establish the existence criterion of the inverse transformation solution for the nonholonomic path.

\section{ACKNOWLEDGEMENT}

This work is supported by the Special Scientific Research Plan of Shaanxi Provincial Department of Education (17JK0048), and the Specialized Research Fund for the Doctor Program of Baoji University of Arts and Sciences (ZK16044).

\section{REFERENCES}

[1] Zhai, J.-Y.; Song, Z.-B. (2018). Adaptive sliding mode trajectory tracking control for wheeled mobile robots, International Journal of Control, 8 pages, doi:10.1080/00207179.2018.1436194

[2] Van Loock, W.; Pipeleers, G.; Diehl, M.; De Schutter, J.; Swevers, J. (2014). Optimal path following for differentially flat robotic systems through a geometric problem formulation, IEEE Transactions on Robotics, Vol. 30, No. 4, 980-985, doi:10.1109/TRO.2014.2305493

[3] Li, L. (2017). Nonholonomic motion planning using trigonometric switch inputs, International Journal of Simulation Modelling, Vol. 16, No. 1, 176-186, doi:10.2507/IJSIMM16(1)CO5

[4] Chung, W.-J.; Nakamura, Y. (2002). Design and control of a chained form manipulator, International Journal of Robotics Research, Vol. 21, No. 5-6, 389-408, doi:10.1177/ $\underline{027836402761393351}$

[5] Nakamura, Y.; Ezaki, H.; Tan, Y.-G.; Chung, W. (2001). Design of steering mechanism and control of nonholonomic trailer systems, IEEE Transactions on Robotics and Automation, Vol. 17, No. 3, 367-374, doi: $10.1109 / 70.938393$

[6] Yamaguchi, H.; Mori, M.; Kawakami, A. (2011). Control of a five-axle, three-steering coupledvehicle system and its experimental verification, IFAC Proceedings Volumes, Vol. 44, No. 1, 12976-12984, doi:10.3182/20110828-6-IT-1002.01455

[7] Yamaguchi, H. (2012). Dynamical analysis of an undulatory wheeled locomotor: a trident steering walker, IFAC Proceedings Volumes, Vol. 45, No. 22, 157-164, doi:10.3182/20120905-3$\underline{\text { HR-2030.00064 }}$ 
[8] Yamaguchi, H. (2007). A path following feedback control law for a trident steering walker, Transactions of the Society of Instrument and Control Engineers, Vol. 43, No. 7, 562-571, doi: $10.9746 / v e . s i c e t r 1965.43 .562$

[9] Dobrin, C.; Bondrea, I.; Pîrvu, B.-C. (2015). Modelling and simulation of collaborative processes in manufacturing, Academic Journal of Manufacturing Engineering, Vol. 13, No. 3, 18-25

[10] Tan, Y.-G.; Li, L.; Liu, M.-Y.; Chen, G.-L. (2012). Design and path planning for controllable underactuated manipulator, International Journal of Advancements in Computing Technology, Vol. 4, No. 2, 212-221, doi:10.4156/ijact.vol4.issue2.26

[11] Li, L.; Tan, Y.-G.; Li, Z. (2014). Nonholonomic motion planning strategy for underactuated manipulator, Journal of Robotics, Vol. 2014, Paper 743857, 10 pages, doi:10.1155/2014/743857

[12] Djedai, H.; Mdouki, R.; Mansouri, Z.; Aouissi, M. (2017). Numerical investigation of threedimensional separation control in an axial compressor cascade, International Journal of Heat and Technology, Vol. 35, No. 3, 657-662, doi:10.18280/ijht.350325

[13] Tan, Y.-G.; Jiang, Z.-Q.; Zhou, Z.-D. (2006). A nonholonomic motion planning and control based on chained form transformation, Proceedings of the 2006 IEEE/RSJ International Conference on Intelligent Robots and Systems, 3149-3153, doi:10.1109/IROS.2006.282337

[14] Pamuk, M. T.; Savaş, A.; Seçgin, Ö.; Arda, E. (2018). Numerical simulation of transient heat transfer in friction-stir welding, International Journal of Heat and Technology, Vol. 36, No. 1, 26-30, doi:10.18280/ijht.360104

[15] Medina, Y. C.; Fonticiella, O. M. C., Morales, O. F. G. (2017). Design and modelation of piping systems by means of use friction factor in the transition turbulent zone, Mathematical Modelling of Engineering Problems, Vol. 4, No. 4, 162-167, doi:10.18280/mmep.040404

[16] Li, Z. X. (1997). A Mathematical Introduction to Robot Manipulation, China Machine Press, Beijing

[17] Sørdalen, O. J. (1993). Conversion of the kinematics of a car with $\mathrm{n}$ trailers into a chained form, Proceedings of the 1993 IEEE International Conference on Robotics and Automation, Vol. 1, 382-387, doi:10.1109/ROBOT.1993.292011 